\title{
International Prognostic Index High Risk Group
}

National Cancer Institute

\section{Source}

National Cancer Institute. International Prognostic Index High Risk Group. NCI

Thesaurus. Code C161809.

A risk group associated with a total score of 4 or 5 on the International Prognostic Index indicating that an individual has a 5 year survival prognosis of $32 \%$. 